\title{
Erratum to: Thoracic and cardiovascular surgery in Japan during 2012: Annual report by The Japanese Association for Thoracic Surgery
}

\author{
Committee for Scientific Affairs, The Japanese Association for Thoracic Surgery • \\ Munetaka Masuda - Hiroyuki Kuwano - Meinoshin Okumura - Jun Amano - Hirokuni Arai • \\ Shunsuke Endo $\cdot$ Yuichiro Doki · Junjiro Kobayashi $\cdot$ Noboru Motomura · Hiroshi Nishida • \\ Yoshikatsu Saiki $\cdot$ Fumihiro Tanaka $\cdot$ Kazuo Tanemoto $\cdot$ Yasushi Toh $\cdot$ Hiroyasu Yokomise
}

Published online: 20 December 2014

(C) The Japanese Association for Thoracic Surgery 2014

\section{Erratum to: Gen Thorac Cardiovasc Surg (2014) \\ 62:734-764 \\ DOI 10.1007/s11748-014-0464-0}

The online version of the original article can be found under doi:10. 1007/s11748-014-0464-0.

Committee for Scientific Affairs, The Japanese Association for Thoracic Surgery, Tokyo, Japan

M. Masuda $(\bowtie)$

Department of Surgery, Yokohama City University, Yokohama, Japan

e-mail: survey-adm@umin.net

\section{H. Kuwano}

Department of General Surgical Science (Surgery I) Gunma

University, Graduate School of Medicine, Gunma, Japan

\section{Okumura}

Department of General Thoracic Surgery, Osaka University

Graduate School of Medicine, Osaka, Japan

\section{J. Amano}

Department of Cardiovascular Surgery, Fujimi-Kogen Medical

Center, Nagano, Japan

\section{H. Arai}

Department of Cardiovascular Surgery, Tokyo Medical and Dental University Graduate School of Medical and Dental

Sciences, Tokyo, Japan

S. Endo

Department of Thoracic Surgery, Jichi Medical University,

Tochigi, Japan

Y. Doki

Department of Gastroenterological Surgery Graduate School

of Medicine, Osaka University, Osaka, Japan
The following errors appeared in the above-cited article.

In 2012 Final report, (A) Cardiovascular surgery section, on the seventh line in the third paragraph, " 14,944 cases in thoracic aortic aneurysm" should read " 15,058 cases in thoracic aortic aneurysm".

\section{J. Kobayashi}

Department of Cardiovascular Surgery, National Cerebral

and Cardiovascular Center, Osaka, Japan

N. Motomura

Department of Cardiovascular Surgery, Toho University, Sakura

Medical Center, Chiba, Japan

\section{H. Nishida}

Department of Cardiovascular Surgery, The Heart Institute of Japan, Tokyo Women's Medical University, Tokyo, Japan

\section{Y. Saiki}

Division of Cardiovascular Surgery, Tohoku University

Graduate School of Medicine, Miyagi, Japan

F. Tanaka

Second Department of Surgery, University of Occupational and Environmental Health, Japan, Fukuoka, Japan

\section{K. Tanemoto}

Department of Cardiovascular Surgery, Kawasaki Medical

School, Okayama, Japan

Y. Toh

Department of Gastroenterological Surgery, National Kyushu

Cancer Center, Fukuoka, Japan

H. Yokomise

Department of General Thoracic Surgery, Faculty of Medicine,

Kagawa University, Kagawa, Japan 
Also in (A) Cardiovascular surgery section, the title for Table 3, "Thoracic aortic aneurysm (total; 14,944)" should read "Thoracic aortic aneurysm (total; 15,058)". (1) "Dissection (total; 6,266") should read (1) "Dissection (total; 6,380"). Additionally, some column totals were incorrect. The corrected table is given below.
In (B) General thoracic surgery section, Table 22, on the eighth line of the table body, "Cardinal reconstruction" should read "Carinal reconstruction". 


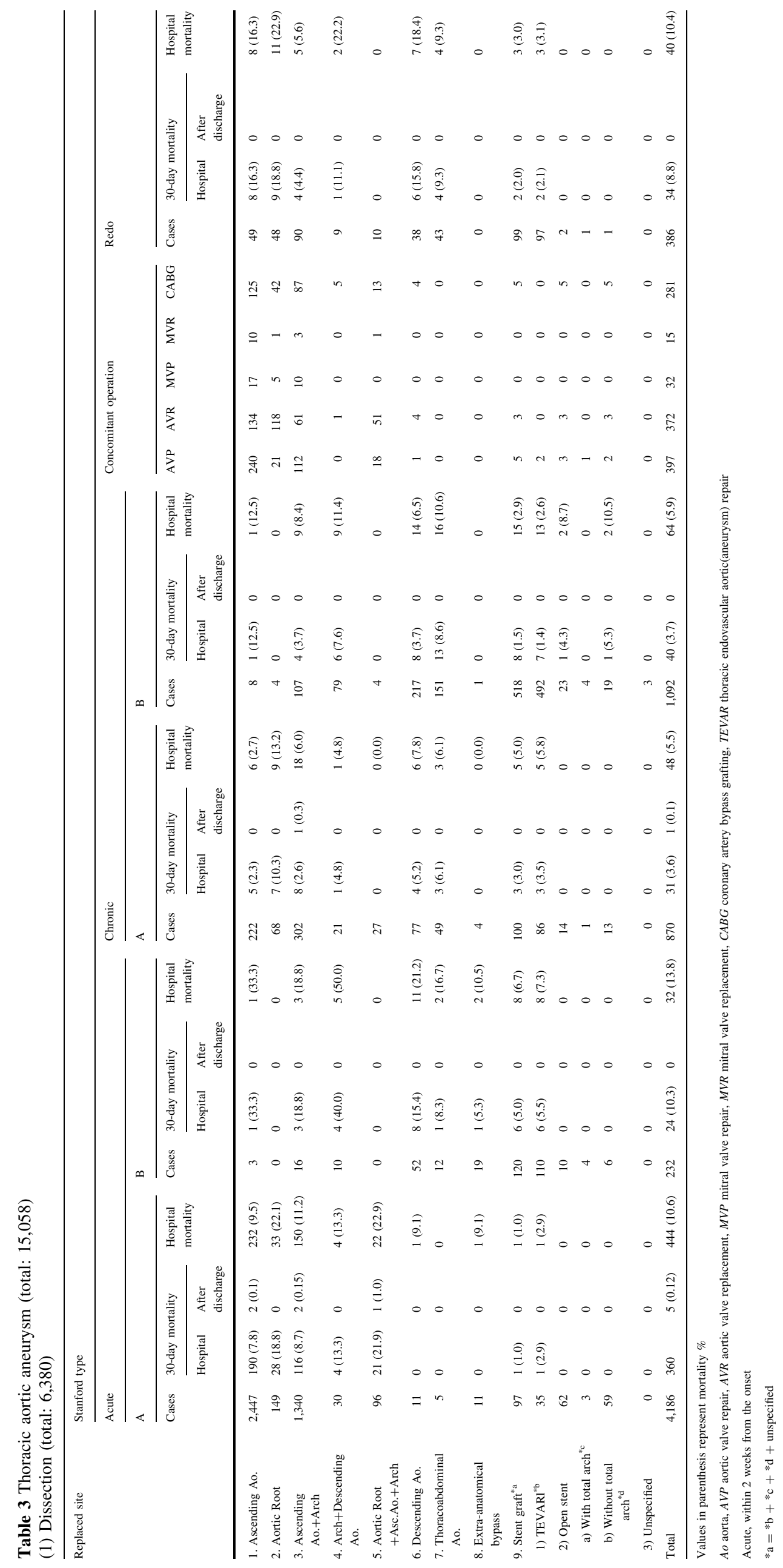

